\title{
LÉGISLATION
}

\section{La Cession des Concessions de Distribution d'Énergie. Commentaire de l'article 33 du cahier type.}

\author{
Par Paul BOUGAULT, Avocat à la Cour d'Appel de Lyon.
}

\author{
L'auteur répond d̀ une question malheureusement bien pratique: Quelle est la portée de l'article 33 ? \\ Permet-il un refus systématique déguisant un piège ou une méchanceté?
}

Texte de l'article 33. - Toute cession partielle ou totale de la concession, tout changement de concessionnaire ne pourront avoir lieu, à peine de déchéance, qu'en vertu d'une autorisation résultant d'une délibération du Conseil municipal approuvée par le Préfet.

Principe. - Remarques applicables aux cessions de toute concession de travaux publics. - Il fallait s'attendre à trouver l'article précité, dans un cahier des charges rédigé par le Ministère des Travaux publics sous le contrôle du Conseil d'Etat. S'il est un principe ancré dans les esprits administratits, c'est que la concession de travaux publics ou de services publics est confiée à une personne jugée capable, par sa compétence technique et sa solvabilité, d'accomplir la tâche qu'elle assume ; en conséquence, la transmission du contrat ne peut être faite qu'en faveur d'une personne remplissant les mêmes conditions ; l'autorité concédante doit s'en assurer et l'affirmer par une délibération clu Conseil Municipal approuvée par le Préfet.

La Cour de Cassation, à propos de conflits entre particuliers, a dû s'occuper de la question; elle l'a jugée sévèrement en annulant des transmissions faites sans que l'autorisation soit intervenue, en estimant que cette absence constituait une nullité radicale et absolue.

Dans l'arrêt du 14 février 1859 (Dalloz 1859, I. 113, af. Mancel contre Jumelais et Pitois), la Chambre civile a statué dans une espèce où l'autorisation administrative avait été refusée ; dans un arrêt du 5 décembre 1882 (Dal. 1883, I, 171, af. Tarbé des Sablons contre faillite des Tramways de Paris à Sèvres), clle a adopté le même système en constatant simplement qu'aucune autorisation préalable n'étạit intervenue ; le 11 février 1884 (Del. 1885, I, 99, af. de Constantin adjudicataire des chemins de fer de Clermont a Tulle contre faillite de la Banque Franco Hollandaise) elle a annulé une cession de concession habilement déguisée sous la vente d'actions de la Compagnie, en employant les mêmes termes que dans les arrêts précédents : il est contraire à l'ordre et à l'intérêt public « que ceux qui ont obtenu une " concession de chemins de fer, puissent, sans le consentement " préalable de l'autorité supérieure la transmettre à des tiers " qui pourraient ne pas offrir les mêmes garanties; que dès lors, " un traité de cession fait sans cette autorisation est entaché "d'une nullité radicale "(1).

(1) C'est surtout les termes dont la Cour a fait usage qui doivent appeler l'attention. Sans doute, tous les arrêts cités sont relatifs à cles Compagnies de chemin de fer qui, pour la plupart, étaient en faillite, et l'on pourrait dire que la nullité de la cession découle
$A u$ Conseil d'Etat, la jurisprudence est identique ; application en est faite non pas à des particuliers évidemment, mais aux justiciables des tribunaux administratifs, c'est-à-dire aux entrepreneurs luttant contre les autorités concédantes ou inversement ; faute d'approbation, le Conseil de Préfeclure ne doit connaître que le concessionnaire primitif et non le concessionnaire substitué (2) ; contre le premier seulement, la déchéance doit être poursuivie (13 juillet 1883, D. 1885, 3. 127), af. Pichard Grison, syndic de la vallee Poussin contre lépartement de la Savoie) et toute procédure doit être dirigée contre lui, sans que ses héritiers puissent protester (arrèt đì 16 mai 1872, Dal. 1873, 3, 2, af. héritiers du sieur Coiret, concessionnaire des eaux de la Ville de Meaux; arrèt du 15 juillet 1894, Dal. 1895, 5, 546, af. Ville de Courthenay contre concessionnaire de la distribution du gaz).

On pourrait citer d'autres décisions administratives on judiciaires. Comme des précédentes, on en extrairait loujours le même principe ; la transmission d'une concession de travaux publics est nulle ct non avenue à l'égard de lous (administration ou contractants) tant qu'elle n'est pas acceptée ; d'une décision du Conseil d'Etat (Compagnie des eaux contre ville de Langres) en date du 20 janvier 1905 (Dal. 1906, 3, 77) rendue célèbre par les conclusions de Monsieur Romieu, il faut appliquer ce principe non seulement à l'aliénation formelle, mais aux simples traités d'exploitation consentis par le concessionnaire à un tiers.

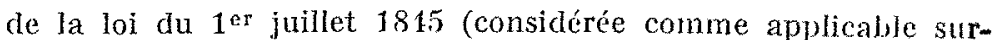
tout en ce qui concerne le litre 7 à tous les chemins de ler d'intérêt général ou local) ; mais les expressions qu'on lit dans les arrêts précités sont susceptibles de s'appliquer à toutes les concessions de travaux ou de services publics. On peut consulter aussi l'arrêt du 14 juillet 1862 (Dal. 1862, I, 518, Compagnie des chemins de fer de Graissessac à Béziers) qui déclare qu'une Compagnie concessionnaire, bien qu'elle ait été mise sous séquestre par l'autorité concédante, peut être déclarée en faillite à la requête de ses créanciers, notamment ses obligataires.

(1) Un arrêt du 31 mai 1878 (Dal. 1878, 3, 62, af. de Méritens) a même refusé à l'acquéreur d'une concession, le droit d'intervenir dans une instance contre le concessionnaire initial : l'annotation de Dalloz fait remarquer que l'intervention est adnise facilement devant la juridiction administrative, mais à la condition qu'elle ait une cause légitime; or, le Conseil a voulu dire que le contrat inexistant vis-à-vis de l'administration ne donnait pas à celui qui en excipait un intérêt valable. Voir sur les conditions suffisantes pour prouver "l'intérêt valable " la décision du Conseil d'Etat : Ville de Langres contre Compagnie départementale des Eaux, 20 janvier 1905 (Dal. 1906, 3, 77) ; la théorie de l'arrêt Méritens paraît bien sévère. 
Limitation de ce principe. - Une règle aussi sévère ne doit pas être exagéréc et nous devons en définir la sphère.

Ellc n'est pas applicable en cas de cession forcée, liquidation judiciaire ou faillite, si ce n'est dans deux occasions, savoir .

a) La très rare hypothèse (arrêt du 23 janvier 1903 , Compagnie d'Electricité de Cognac, Ville de Cognac, D. 1904, 3, 61), dans laquelle le cahier des charges comporte une clause très formelle et draconnienne, considérant la faillite comme une cause de résiliation immédiate. b) Celle où la faillite a suspendu le cours du service public (arrêt du 22 mars 1902, D.1903, 3, 87, af. Chambon et Guébin, liquidateurs du concessionnaire contre commune de Bric-Comte-Robert).

En dehors de ces cas, le Conseil d'Etat, pour éviter l'injustice consistant à dépouiller les créanciers du meilleur de leur gage, a interprété les clauses du cahier des charges d'une façon libérale. Le concessionnaire du gaz de la ville de Saint-Girons avait dans son cahier des charges la phrase suivante : «Si, pendant " le cours de la concession, le concessionnaire venait à inter" rompre son exploitation, ou était hors d'état de la continuer, il " serail déchu de plein droit de sa concession et la ville repren" drait le matériel et pourvoirait au service public par tel moyen " qu'elle jugcrait convenable ". La faillite étant survenue, mais le syndic continuant l'exploitation, il a été jugé que la commune n'avait pas, en s'abstenant d'approuver le successeur, le droit dé paralyser une exploitation qui était convenablement reprise et maintenue (arrêt du 23 février 1883, D. 1884, 3, 101, af. Boué)

$2^{\circ}$ Il en serail certainement de mème, à notre avis, si le Conseil d'Etat se trouvait en présence de la succession du concessionnaire au licu de se trouver en présence de son syndic. Pourvu que les héritiers s'entendent pour proposer un remplaçant capable, présentant toutes les garanties, le Conseil municipal, par une obstination méchante ou même systématique, par des exigences portant sur des modilications de tarifs ou des avantages nouveaux, ne saurait porter préjudice à une succession tout entière en refusant le remplaçant. La raison de décider ainsi est double : d'abord les concessions sont généralement fort longues, trente ou quarante ans el lorsque le concessionnaire n'est plus de la première jeunesse, la commune doit sç rendre compte que jamais n'est plus vraie la présomption écrite dans l'article 1122 du Code civil : "On est consé avoir stipulé pour soi el ses héritiers ou " ayants cause"; ensuite le Conseil d'Etat aime à statuer "d'après " l'interprélation que les parties ont dû donner elles-mèmes à "leur acte"; or, il n’a pu entrer, même dans l'esprit de la commune concéflante, que le concessionnaire mènerait son entreprise jusqu'à un terme si éloigné sans avoir à redouter ni décès, ni maladie grave pouvant interrompre l'œuvre accomplie.

$3^{\circ}$ Il faut encore tenir compte que, dans un arrêt relatifvement récent (Vuillaüme contre commune de Trois-Villes, 13 novembre 1908, Revue des Concessions, année 1909, page 82) qui a toute la portée d'une décision doctrinale, le Conseil d'Etat a condamné le refus méchant et sans cause dont des concessionnaires gaziers, les sieurs Vuillaume, avaient été victimes dans les circonstances suivantes : par un article de leur cahier des charges, ces concessionnaires s'étaient réservé le droit de céder à des tiers le bénéfice de leur contrat, sous la condition que les nouveaux concessionnaires aient été au préalable agréés par la commune ; le 10 octobre 1902, ils cèdent à la Société anonyme Internationale d'Acétylène leur exploitation, en préviennent le Conseil qui de- mạnde le nom des associés ; plus de deux ans après, le 21 janvier 1905, la commune signifie un refus non motivé au Conseil Municipal ; cet acte de refus a été considéré par le Conseil de Préfecture comme valable, mais le Conseil d'Etat a cassé l'arrêté et il a décleré que l'usage que la commune avait fait de la clause précitée n'avait pu entrer dans l'intention des parties et avait jeté, dans l'exécution du contrat, un trouble qui rendait les sieurs Vuillaume aptes à demander la résillation du marché ; il a accordé la résiliation du contrat aux sieurs Vuillaume qui la demandaient et s'il ne leur a pas alloué d'indemnité, c'est que ceux-ci avaient eu le tort de se faire justice eux-mêmes, en abandonnant volontairement leur exploitation.

40 Il faut aller plus loin, à notre avis, et dans le cas de refus motivé sur des prétextes et non sur des raisons sérieuses, on ne devrait pas hésiter à demander à la juridiction administrative d'apprécier ce refus, d'en examiner la valeur intrinsèque et de l'annuler si vraiment il cache un marchandage.

Le véritable aspect de la question est celui-ci ; dans la très grande majorité des cas, la commune n'oppose une résistance obstinée que dans le but de faire une pression sur le concessionnaire substitué, afin que celui-ci consente à acquitter une sorte de droit de bienvenue, en acceptant des sujétions non prévues au contrat. Ce n'est pas pour créer ces sortes de marchandages que le Conseil Municipal détient les pouvoirs dont il est investi par la loi et qui paraissent limités à la surveillance du service dont il est moralement responsable vis-à-vis du public ; le refus devrait être maintenu ou annulé, selon qu'il est sincère ou méchamment allégué.

$5^{\circ}$ Enfin, la transmission, sans acceptation de la part de la commune, n'autorise pas celle-ci à se mettre en possession de l'exploitation et d'en assurer le service, pas plus d'ailleurs que de réclamer des dommages-intérêts (1). Tout ce que la commune peut envisager, c'est la déchéance du concessionnaire, soit que le Maire puisse la prononcer dans le cas très rare où le contrat lui en donne le droit formel, soit, dans le cas contraire, que la commune doive la demander au Conseil de Préfecture; mais " demander " ne signifie pas obtenir.

Si donc, l'unique sanction, la déchéance, doit être soumise à l'appréciation raisonnée du juge, n'en découle-t-il pas que la même appréciation doit intervenir au sujet du fait qui y donne lieu et que si la transmission ne faisait courir aucun danger at service, elle ne saurail être arbitrairement refusée ?

(1) On est vraiment étonnée de la facilité avec lacquelle les communes se persuadent que la déchéance est pour elles un moyen très facile d'obtenir le droit de' s'enrichir gratuitenient au préjudice du concessionnaire, en prenant, sans autre forme de procès, tout ce que celui-ci a édifié et en considérant cette prise de possession comme le moyen d'avoir des dommages-intérêts, en compensation de la déchéance. C'est un point de vue absolument faux: le résultat certain de la déchéance est de faire perdre au concessionnaire la totalité de son cautionnement ou au moins la partie qui ne lui a pas encore été restituée ; mais en cas de déchéance encourue, le concessionnaire a droit au prix des travaux exécutés avant la déchéance, à moins que le cahier des charges ne renferme une clause absolument contraire, car la disposition du cahier des charges qui stipule la dechéance, comme clause pénale, en cas d'inexécution des obligations du concessionnaire, exclut tout autre pénalité et ne permet pas à l'administration de réclamer des dommages-intérêts dans les conditions du droit civil. 